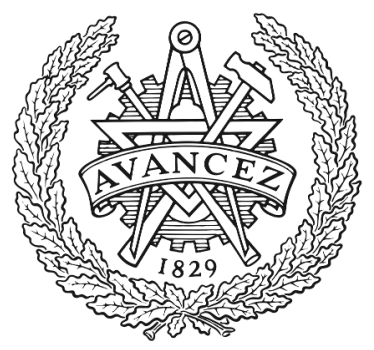

CHALMERS

UNIVERSITY OF TECHNOLOGY

\title{
Period multiplication in a parametrically driven superconducting resonator
}

Downloaded from: https://research.chalmers.se, 2023-04-26 01:29 UTC

Citation for the original published paper (version of record):

Svensson, I., Bengtsson, A., Bylander, J. et al (2018). Period multiplication in a parametrically driven superconducting resonator. Applied Physics Letters, 113(2). http://dx.doi.org/10.1063/1.5026974

N.B. When citing this work, cite the original published paper. 


\title{
Period multiplication in a parametrically driven superconducting resonator
}

\author{
Ida-Maria Svensson, ${ }^{\text {a) }}$ Andreas Bengtsson, Jonas Bylander, Vitaly Shumeiko, \\ and Per Delsing ${ }^{\text {b) }}$ \\ Microtechnology and Nanoscience, Chalmers University of Technology, SE-41296 Göteborg, Sweden
}

(Received 26 February 2018; accepted 22 June 2018; published online 9 July 2018)

\begin{abstract}
We report on the experimental observation of period multiplication in parametrically driven tunable superconducting resonators. We modulate the magnetic flux through a superconducting quantum interference device, attached to a quarter-wavelength resonator, with frequencies $n \omega$ close to multiples, $n=2,3,4$, and 5, of the resonator fundamental mode and observe intense output radiation at $\omega$. The output field manifests $n$-fold degeneracy with respect to the phase, and the $n$ states are phase shifted by $2 \pi / n$ with respect to each other. Our demonstration verifies the theoretical prediction by Guo et al. [Phys. Rev. Lett. 111, 205303 (2013)] and paves the way for engineering complex macroscopic quantum cat states with microwave photons. Published by AIP Publishing.

https://doi.org/10.1063/1.5026974
\end{abstract}

The technology of circuit quantum electrodynamics ${ }^{1,2}$ offers an excellent platform for observation and exploration of parametric oscillation phenomena in the quantum domain. By connecting Josephson elements to superconducting resonators, one is able to induce the nonlinearity of the electromagnetic field and realise temporal high frequency control of the resonator parameters. ${ }^{3-5}$ This in combination with high quality factors of the superconducting resonators makes the parametric oscillation regime, above the instability threshold, easily accessible with relatively small modulation intensities. Furthermore, low temperatures in the range of 10 $\mathrm{mK}$ allow us to investigate the quantum properties of the oscillator states. Using this technique, both the degenerate ${ }^{6}$ (pumping at twice a resonator mode frequency) and nondegenerate $^{7}$ (pumping at the sum of two resonator mode frequencies) parametric oscillations have been experimentally investigated.

An inherent property of parametric oscillations is phase degeneracy of the oscillator states. The non-degenerate oscillator has a continuous phase degeneracy, ${ }^{7-9}$ while the degenerate oscillator exhibits a discrete, two-fold degeneracy, which is manifested by two correlated $\pi$-shifted steady states. ${ }^{6,10}$ In the quantum regime, these states form coherent superpositions of optical coherent states, cat states, ${ }^{11,12}$ which can be used as building blocks for a photonic quantum processor. ${ }^{13}$

Quantum properties of the degenerate parametric oscillations motivate great interest in finding ways to engineer more complex multiply degenerate oscillator states. The period multiplication phenomenon in nonlinear oscillators $^{14,15}$ offers an attractive approach to the problem. In recent papers, an experimental demonstration of the period tripling in a superconducting resonator was reported, ${ }^{16}$ and quantum properties of the emerging three-fold degenerate state were theoretically investigated. ${ }^{17}$ In that experiment, the self-sustained oscillations of the resonator mode were excited by injecting an external signal with a frequency close to three times the fundamental mode frequency.

\footnotetext{
a)ida-maria.svensson@chalmers.se

b)per.delsing@chalmers.se
}

In this letter, we report an experimental demonstration of a period multiplication phenomenon-generation of oscillations with multiples, $n=2,3,4$, and 5, of the pumping field period. In this experiment, we employ a different method of parametric excitation-temporal modulation of magnetic flux through a superconducting quantum interference device (SQUID) attached to the resonator. We modulate the flux with frequencies $n \omega$, close to the multiples of the fundamental mode frequency, and observe emergence of $n$-fold phase degenerate self-sustained oscillations with frequency $\omega$. A similar method has been used earlier to demonstrate degenerate parametric oscillations ${ }^{6}$ - the most known form of the period multiplication with $n=2$. However, for $n>2$, the flux modulation results in modulation of the resonator nonlinearity rather than frequency, which leads to qualitatively different properties of the oscillations.

Quantum effects under parametric modulation of a nonlinearity have been theoretically investigated in Ref. 18. Our observations demonstrate a practical realization of the model introduced there. The connection can be seen from the form of the differential inductance to the SQUID that provides tunability and nonlinearity of the resonator

$$
L_{S Q}(\phi)=\frac{L_{S Q, 0}}{\cos (f / 2) \cos \phi-\left(E_{-} / E_{+}\right) \sin (f / 2) \sin \phi} .
$$

Here, $f(t)=2 \pi \Phi(t) / \Phi_{0}$ is a normalized applied magnetic flux, $\Phi_{0}=h / 2 e$ is the flux quantum, $E_{ \pm}=\left(E_{J 1} \pm E_{J 2}\right) / 2$ are the combinations of Josephson energies of the two SQUID junctions, and $L_{S Q, 0}=(\hbar / 2 e)^{2}\left(1 / 2 E_{+}\right)$is the SQUID inductance at zero flux. The modulation of the magnetic flux affects not only the quadratic term in the Taylor expansion of the inductance over the phase, $\phi$, i.e., the resonator frequency, but also all the higher order nonlinear terms. By choosing a proper modulation frequency, one can selectively address any of these nonlinear terms, thus implementing the period multiplication regime considered in Ref. 18.

In our experiment, we use a frequency-tunable, quarterwavelength coplanar waveguide microwave resonator. ${ }^{3-5}$ The resonator is capacitively coupled to a transmission line 
TABLE I. Resonator parameters for the samples used to produce the datasets presented in this paper. $d$ is the resonator length, $\omega_{1}(0)$ is the resonance frequency of the first mode at zero flux, $I_{c}$ is the SQUID critical current, $C_{S Q}$ is the SQUID capacitance, $\gamma_{0}=L_{S Q, 0} / L_{0} d$ is the inductive participation ratio, $L_{0}$ and $C_{0}$ are the inductance and capacitance per unit length of the coplanar waveguide transmission line, respectively, $3 \omega_{1}(0)-\omega_{2}(0)$ is the spectrum anharmonicity, $2 \Gamma_{1}$ is the resonator damping rate at zero flux, and $Q_{c, 1}(0)$ and $Q_{l, 1}(0)$ are the coupling and loaded quality factors for the first mode at zero flux. Sample \#3 has not been fully characterized but has a very similar design except a slightly lower $\gamma_{0}$ due to the longer length.

\begin{tabular}{|c|c|c|c|c|c|c|c|c|c|c|c|}
\hline Sample & $\mathrm{d}(\mathrm{mm})$ & $\omega_{1} / 2 \pi(\mathrm{GHz})$ & $I_{c}(\mu \mathrm{A})$ & $C_{S Q}(\mathrm{fF})$ & $\gamma_{0}(\%)$ & $L_{0}(\mu \mathrm{H} / \mathrm{m})$ & $C_{0}(\mathrm{nF} / \mathrm{m})$ & {$\left[3 \omega_{1}(0)-\omega_{2}(0)\right] / 2 \pi(\mathrm{MHz})$} & $2 \Gamma_{1}(0) / 2 \pi(\mathrm{MHz})$ & $Q_{c, 1}(0)\left(10^{3}\right)$ & $Q_{l, 1}(0)\left(10^{3}\right)$ \\
\hline$\# 1$ & 5.2 & 5.225 & 1.47 & 63 & 10.7 & 0.41 & 0.17 & 86 & 0.49 & 11.4 & 10.7 \\
\hline$\# 2$ & 5.2 & 5.504 & 1.90 & 86 & 7.7 & 0.44 & 0.16 & 136 & 0.38 & 19 & 14.5 \\
\hline \#3 & 6.8 & 4.035 & & & & & & 21 & & & \\
\hline
\end{tabular}

at one end and grounded at the other end via a SQUID. The frequency spectrum of such a resonator is non-equidistant, which allows selective parametric excitation of individual modes. Several devices have been investigated, and all of them exhibit a qualitatively similar behavior. Technical data for the three samples used to produce the data are presented in Table I. Although the SQUIDs are designed to be symmetric, i.e., equal junctions, there can be variations in the fabrication process. Up to a few percent variation in critical current can appear.

The samples are fabricated using standard microfabrication techniques. The circuits are etched in a thin niobium film on c-plane sapphire substrates. The SQUIDs are made of aluminium and fabricated using two-angle evaporation. The measurements are performed at low temperatures, $\hbar \omega \gg k_{B} T$, in a dilution cryostat, where the base temperature is around $12 \mathrm{mK}$. Our measurement setup is sketched in Fig. 1. We use two input lines, one for an external probe signal and one for flux modulation. Both are attenuated to reduce thermal noise. A static magnetic field is applied using a superconducting coil, mounted close to the sample.

The sample is measured using a reflection setup. A directional coupler is employed to route the signal, and two

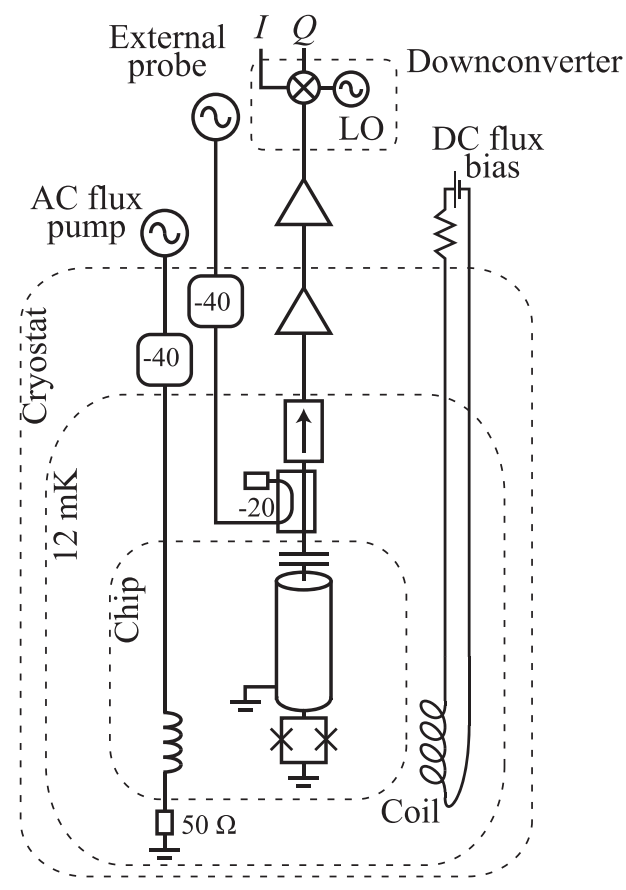

FIG. 1. Schematic of the measurement setup. The resonator can be excited via an external probe or via the flux pump line. The chip is mounted in a sample box at the mixing chamber stage of a dilution cryostat with a base temperature of $12 \mathrm{mK}$. Measurements are done using heterodyne detection. isolators protect the resonator from amplifier noise. The output signal is amplified by a cryogenic amplifier and then further boosted by a room temperature amplifier. The signal undergoes heterodyne down-conversion, and the in- and out-of-phase quadrature voltages, $I(t)$ and $Q(t)$, are then sampled in a digitizer.

Our measurement frequency is placed close to the fundamental mode, $\omega=\omega_{1}+\delta$, and a microwave tone is applied to the flux pump line at a frequency $n \omega, n=2,3,4$, and 5 . For a small pump intensity, we only detect noise, while when the pump intensity exceeds a certain threshold, a strong output signal emerges indicating excitation of the parametric oscillator, Fig. 2. The oscillations are observed in a wide range of the biasing flux controlling the mode frequency, including zero flux for odd multiples, for even multiples no period multiplication is observed at zero flux. The regions of the pump power and frequency, where the oscillations are observed, strongly vary with $n$. As a rule, excitation of odd multiples requires smaller power than the even ones. The oscillations appear at small red detuning from resonance and reach far into the low frequency region, similar to the parametric oscillations ${ }^{7}$ and the period tripling oscillations under current injection. ${ }^{16}$ The histograms in Fig. 2 were measured at detunings of between -1.5 and $-18 \mathrm{MHz}, 3-40$ times the resonator bandwidth.

We analyze the output by sampling the quadratures and produce the histograms shown in Fig. 2. The bright spots in the histograms correspond to steady states of the oscillator; they indicate a discrete degeneracy of the oscillator states. The plots in the right column of Fig. 2 show spots that are symmetrically displaced from the origin, i.e., have equal intensities, $P=I^{2}+Q^{2}$, phase shifted by $2 \pi / n$ with respect to each other. Overall orientations of the multiplets are defined by the pump signal phase, which we do not control in this experiment. Hence, the orientations of different histograms are random.

The right column histograms of Fig. 2 were measured in the well-established oscillator regime far away from the boundaries of the oscillation visibility. Those to the left were measured closer to the boundaries, and here, we also see a central spot representing the oscillator ground state. This is a multistability region, which is typical for microwave parametric oscillations, as explained theoretically ${ }^{10,16}$ and observed experimentally for the degenerate parametric oscillator ${ }^{6}$ and for the period-tripling oscillator. ${ }^{16}$ The multistability is explained by the coexistence of the stable excited states of the oscillator and the oscillator ground state. In histograms (a) and (e), the excited states are not well resolved. 

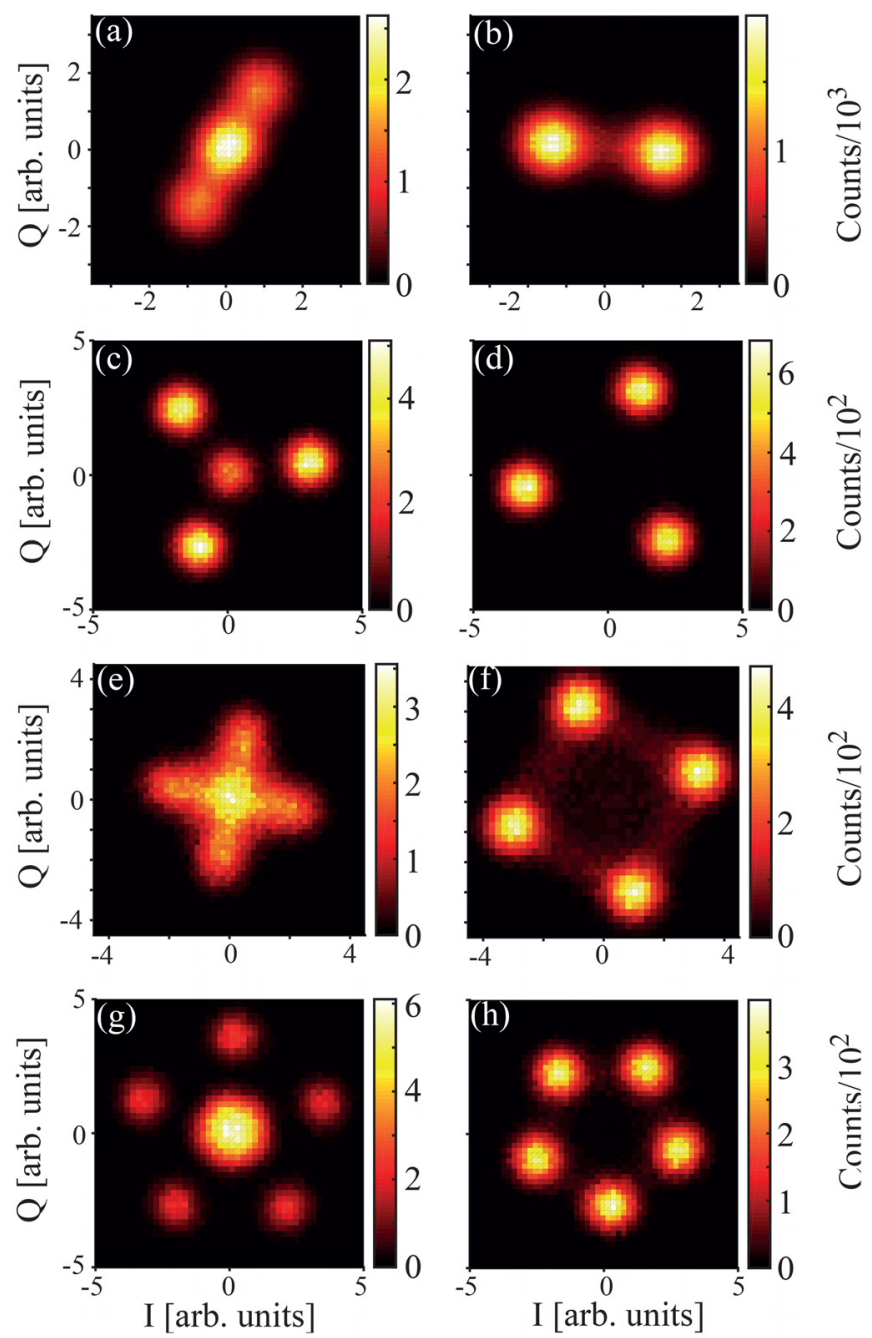

FIG. 2. Quadrature histograms of period multiplication oscillations generated by applying a microwave signal to the flux pump line at multiples $n$ of the fundamental mode frequency. (a) and (b) Sample \# 1, $n=2, \Phi=0.1 \Phi_{0}$ and detuning $\delta / 2 \pi=-2$ and $-1.5 \mathrm{MHz}$; (c), (d) sample \#1, $n=3, \Phi=0.17$ $\Phi_{0}$ and detuning $\delta / 2 \pi=-7$ and $-9 \mathrm{MHz}$; (e) and (f) sample \#3, $n=4, \Phi$ $=0.07 \Phi_{0}$ and detuning $\delta / 2 \pi=-9$ and $-18 \mathrm{MHz}$; (g) and (h) sample \#1, $n=5, \Phi=0.1 \Phi_{0}$ and detuning $\delta / 2 \pi=-7$ and $-4 \mathrm{MHz}$. The output signals correspond to around 60 photons.

This can be explained by large critical fluctuations around emerging coherent oscillations having a small intensity. In histogram (f), faint lines between the bright spots are visible. These lines reveal the existence of jumps, i.e., switching between the oscillator steady states. Indeed, the data points represent the quadrature values averaged over the measurement time intervals. If no jump occurs during the measurement, the result falls in one of the steady state spots; however, if a jump occurs during the measurement, the averaging places the point between the spots. Thus, the presence of the lines in histogram (f) indicates a switching rate exceeding the measurement sampling rate, which in this particular case was $100 \mathrm{kHz}$. This is the sampling rate used for all the datasets except those in Figs. 2(a) and 2(b), which are sampled at $50 \mathrm{kHz}$.

To explain our observations, we analyze the multimode quantum Hamiltonian of the resonator ${ }^{10}$

$$
H=\sum_{n} \hbar \omega_{n} \hat{a}_{n}^{\dagger} \hat{a}_{n}+V(\hat{\phi}, t),
$$

where $\omega_{n}$ is the frequency of the $n$-th eigenmode, $\hat{a}_{n}$ and $\hat{a}_{n}^{\dagger}$ are the mode annihilation and creation operators, and the sum goes over all resonator modes. The variable $\hat{\phi}(t)$ refers to a dynamic deviation from the static value, $\tan \phi_{0}$ $=-\left(E_{-} / E_{+}\right) \tan (F / 2)$, of the phase at the SQUIDterminated edge of the resonator, $\hat{\phi}(x=d, t)=\phi_{0}+\hat{\phi}(t)$. Here, $F=2 \pi \Phi_{d c} / \Phi_{0}$ indicates a normalized static magnetic flux, and $d$ denotes the resonator length. An expansion of $\hat{\phi}$ over the cavity modes reads

$$
\hat{\phi}=\sum_{n} \beta_{n}\left(\hat{a}_{n}+\hat{a}_{n}^{\dagger}\right), \quad \beta_{n}=\gamma \sqrt{\frac{8 \pi Z_{0} k_{n} d}{R_{K}}},
$$

where $Z_{0}=\sqrt{L_{0} / C_{0}}, R_{K}=h / e^{2}$, and $k_{n}=\omega_{n} / v$ is the mode eigenvector defined by the spectral equation, ${ }^{10}$ $k_{n} d \tan \left(k_{n} d\right)=1 / \gamma$, where $\gamma=E_{L, c a v} /\left(2 E_{+} \cos (F / 2)\right) \ll 1$ is the participation ratio of the SQUID inductance versus the cavity inductance. Here, $L_{0}$ and $C_{0}$ are the inductance and capacitance per unit length of the coplanar waveguide transmission line, respectively. Together they define the phase velocity in the waveguide resonator, $v=1 / \sqrt{L_{0} C_{0}}$. The potential energy $V(\hat{\phi}, t)$ in Eq. (2) represents the nonlinear part of the inductive energy of the SQUID and has the form for an asymmetric SQUID,

$$
\begin{aligned}
V(\hat{\phi}, t) \approx & -E_{+} \cos (F / 2)\left(\cos \hat{\phi}+\frac{\hat{\phi}^{2}}{2}\right) \\
& +\delta f(t)\left[E_{+} \sin (F / 2) \cos \hat{\phi}+\frac{E_{-}}{\cos (F / 2)} \sin \hat{\phi}\right],
\end{aligned}
$$

where $\delta f(t)=2 \pi \Phi_{a c}(t) / \Phi_{0}$ describes the pump, i.e., a small-amplitude temporal modulation of the applied magnetic flux, here considered in the linear approximation. Other adopted approximations in Eq. (4) include the assumption of a small SQUID asymmetry, $E_{-} \ll E_{+}$, and a negligibly small effect of the SQUID capacitance.

It is appropriate to make the following comments to Eq. (4): (i) the symmetric part of the potential, $\propto E_{+}$, contains only even powers of $\hat{\phi}$, which implies that only even order subharmonics can be excited by the flux pump if the SQUID is perfectly symmetric. (ii) The asymmetric part, $\propto E_{-}$, is responsible for the excitation of odd order subharmonics; this part, however, also contains a linear term, $\propto \hat{\phi}$, which implies that the nonstationary magnetic flux directly generates a field inside the resonator. The effect of this field is similar to the one created by externally applied current drive investigated in Ref. 16. Thus, the flux modulation method allows for excitation of both even and odd subharmonics. In the latter case, a secondary current drive effect complements the flux pump [see below in Eq. (7)]. We note that the excitation of even order period multiples is not possible by current driving due to symmetry constraint.

Suppose the magnetic flux is modulated with a frequency $n \omega=n\left(\omega_{1}+\delta\right)$ and $\delta f(t)=\delta f_{0} \cos n \omega t$. Then, we assume, focusing on the resonant response of the fundamental resonator mode $n=1$, the field in the cavity to be a superposition of two harmonics,

$$
\hat{\phi}(t)=\beta_{1} \hat{a}_{1}(t) e^{-i \omega t}+\beta_{n} \hat{a}_{n}(t) e^{-i n \omega t}+\text { H.c. },
$$


where $\hat{a}_{n}$ is the response of a mode with a frequency close to the pump frequency (the term is only needed for odd $n$ ).

Following the method of Refs. 10 and 16, we derive a shortened dynamical equation for a slowly varying amplitude $\hat{a}_{1}(t)$ in the rotating wave approximation. This equation has a universal form for all integers $n$,

$$
i \dot{\hat{a}}_{1}+\left(\delta+i \Gamma_{1}+\alpha \hat{a}_{1}^{\dagger} \hat{a}_{1}\right) \hat{a}_{1}+\epsilon_{n}\left(\hat{a}_{1}^{\dagger}\right)^{n-1}=\sqrt{2 \Gamma_{1}} \hat{a}_{1}^{i n}
$$

and essentially coincides with the model equation introduced in Ref. 18. Here, $\Gamma_{1}$ is the resonator damping, $\hat{a}_{1}^{\text {in }}$ indicates the environmental noise, and the coefficients, $\alpha=\left(E_{+} / \hbar\right)$ $\cos (F / 2) \beta_{1}^{4}$ and $\epsilon_{2}=\left(E_{+} / \hbar\right) \cos (F / 2) \beta_{1}^{2} \delta f_{0}$, are familiar from the study of degenerate parametric resonance. ${ }^{10}$ The pump coefficient for period quadrupling, $\epsilon_{4}$, is expressed through $\epsilon_{2}$ and has the form $\epsilon_{4}=-\epsilon_{2}\left(\beta_{1}^{2} / 2\right)$. Similar scaling holds for higher even-order coefficients, $\epsilon_{2 k} \propto \epsilon_{2} \beta_{1}^{2 k-2}$. The pump coefficient for period tripling consists of two contributions,

$$
\epsilon_{3}=\frac{E_{-}}{2 \hbar} \frac{\beta_{1}^{3} \delta f_{0}}{\cos (F / 2)}+\frac{E_{+}}{\hbar} \cos (F / 2) \beta_{1}^{2} \beta_{3} a_{3} .
$$

The first term is the direct flux pump effect, and the second term describes the secondary, current-drive effect. The latter term may also include some spurious external field effects, e.g., due to a cross talk between the flux line and the resonator. All higher odd-order coefficients have a similar structure with the overall scaling factor $\beta_{1}^{2 k-2}$.

Period multiplying oscillations have large amplitude far from the thresholds and are described with quasiclassical solutions to Eq. (6). The stationary solution for a given $n$ has the form $a_{1, n}=r_{n} e^{i \theta_{n}}$, with $\sin \left(n \theta_{n}-\arg \epsilon_{n}\right)=\Gamma_{1} /|\epsilon| r_{n}^{n-2}$. Such solution has an $n$-fold degeneracy, as seen in Fig. 2, with phases $\theta_{n}=\theta_{n, 0}+2 \pi m / n(m=0, \ldots, n-1)$. The reference phase $\theta_{n, 0}$ depends on the phase of the pump and the parameters of the working point.

The outlined calculation qualitatively explains our experimental observations - the period multiplication effect, degeneracy, and phase symmetry of the oscillations, including the dependence of effective pump strength on $n$. It also relates the heuristic coefficients of the model ${ }^{18}$ to the device parameters. A detailed quantitative analysis of the data is beyond the scope of this paper since it requires a theoretical advance in construction and stability analysis of the oscillatory solutions.

The nonlinear parametrically driven resonator has indeed a very rich behavior. Further insight into the properties of period multiplication can be gained by exploring regimes beyond the observed steady-state multiplets. In Fig. 3 , we present a histogram of the period-tripling oscillations measured at larger red detuning, where the oscillation intensity is substantially stronger than in Fig. 2. Here, we detect coexistence of two triplet states, with different amplitudes and different orientations, presumably corresponding to a higher order nonlinearity.

Injection of a weak probe signal into the resonator is known to produce a phase-locking effect, observed, for example, in the degenerate parametric oscillator. ${ }^{19,20}$ There, the effect was manifested by a gradual disappearance of one
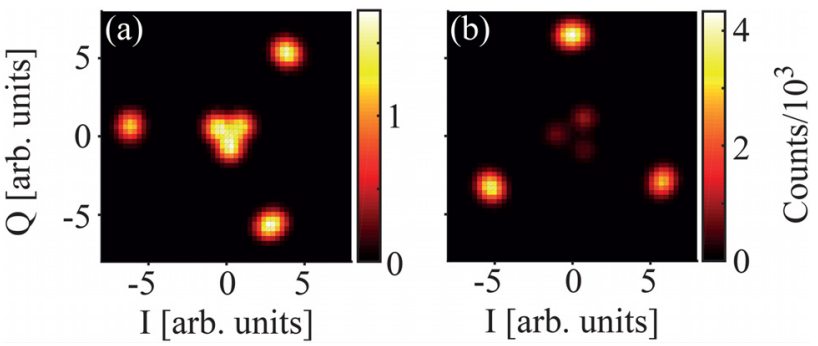

FIG. 3. Histograms of period-tripling oscillations at larger output signal levels, revealing an additional triplet of excited states with amplitude and orientation different from the main triplet. The difference between panels (a) and (b) is that the latter is measured at $1 \mathrm{~dB}$ higher pump power. Sample \#1 was used.

of the doublet states and explained by a symmetry breakdown under on-resonance injection. Similarly, in the period tripling regime, we observe a gradual disappearance of the triplet components when an on-resonance probe signal is applied, see Figs. 4(a) and 4(b). Phase locking has also been observed in a non-degenerate parametric oscillator ${ }^{7,8}$ manifested by a suppressed phase diffusion. The phase locking is a common effect in oscillators with phase degeneracy, and it can be visualized as a tilt of a quasiclassical metapotential of the oscillator by a driving term, $\sim F^{*} a+F a^{\dagger}$.

Furthermore, injection of a probe signal slightly detuned, by $1 \mathrm{~Hz}$, produces a completely different, dramatic effect on the period tripling oscillations, see Figs. 4(c) and 4(d). Instead of breaking the symmetry of the overall triplet pattern, the individual round spots deform into crescents whose size increases with increasing probe intensity. The behavior resembles the phase locking effect observed in nondegenerate parametric oscillators, ${ }^{7,9}$ where the oscillator state possesses continuous phase degeneracy. This resemblance leads us to interpret our observation as a result of a deformation of each stable stationary state of the triplet into a stable cycle with simultaneous phase locking. A further theoretical investigation is required to quantitatively explain these observations.
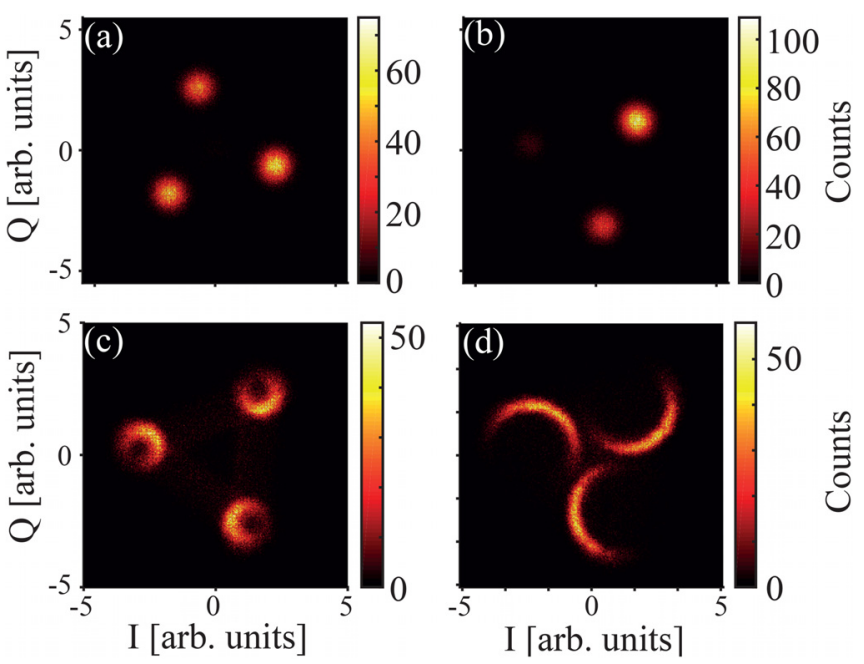

FIG. 4. Histograms of output oscillations affected by a probe signal. (a) and (b) Phase locking effect of an on-resonance probe, $20 \mathrm{~dB}$ stronger in (b) than in (a). (c) and (d) Effect of a $1 \mathrm{~Hz}$ detuned probe signal, $10 \mathrm{~dB}$ stronger in (d) than in (c). Sample \#2 was used. 
In conclusion, we observed the period-multiplication phenomena in parametrically driven superconducting resonators. We observed robust output radiation at a frequency close to the fundamental resonator mode, with $n=2,3,4$, and 5 evenly shifted phase components under an applied pump signal with frequencies $n$ times the detection frequency. Our qualitative analysis of the resonator dynamics agrees with the observations and corroborates the model proposed in Ref. 18. Demonstration of multiply degenerate classical states under period multiplication suggests a possibility, in analogy with the double degeneracy of parametric oscillations, ${ }^{12}$ of engineering of multicomponent quantum superpositions of macroscopic coherent states-multicomponent cat states. ${ }^{13}$ Our observations call for further exploration of the quantum properties of the period-multiplication and a search for engineering methods of macroscopic quantum states in this regime.

We gratefully acknowledge financial support from the Wallenberg Foundation, the Swedish Research Council, and the European Research Council. J.B. acknowledges partial support by the EU under REA Grant Agreement No. CIG618353.

${ }^{1}$ R. J. Schoelkopf and S. M. Girvin, "Wiring up quantum systems," Nature 451(7179), 664-669 (2008).

${ }^{2}$ X. Gu, A. F. Kockum, A. Miranowicz, Y.-X. Liu, and F. Nori, "Microwave photonics with superconducting quantum circuits," Phys. Rep. 718-719, 1-102 (2017).

${ }^{3}$ M. Wallquist, V. S. Shumeiko, and G. Wendin, "Selective coupling of superconducting charge qubits mediated by a tunable stripline cavity," Phys. Rev. B 74(22), 224506 (2006).

${ }^{4}$ M. Sandberg, C. M. Wilson, F. Persson, T. Bauch, G. Johansson, V. Schumeiko, T. Duty, and P. Delsing, "Tuning the field in a microwave resonator faster than the photon lifetime," Appl. Phys. Lett. 92, 203501 (2008).

${ }^{5}$ A. Palacios-Laloy, F. Nguyen, F. Mallet, P. Bertet, D. Vion, and D. Esteve, "Tunable resonators for quantum circuits," J. Low Temp. Phys. 151(3), 1034 (2008).
${ }^{6}$ C. M. Wilson, T. Duty, M. Sandberg, F. Persson, V. Shumeiko, and P. Delsing, "Photon generation in an electromagnetic cavity with a timedependent boundary," Phys. Rev. Lett. 105(23), 233907 (2010).

${ }^{7}$ A. Bengtsson, P. Krantz, M. Simoen, I.-M. Svensson, B. Schneider, V. Shumeiko, P. Delsing, and J. Bylander, "Nondegenerate parametric oscillations in a tunable superconducting resonator," Phys. Rev. B 97, 144502 (2018).

${ }^{8} \mathrm{~W}$. Wustmann and V. Shumeiko, "Nondegenerate parametric resonance in a tunable superconducting cavity," Phys. Rev. Appl. 8(2), 024018 (2017).

${ }^{9}$ F. Sun, X. Dong, J. Zou, M. I. Dykman, and H. B. Chan, "Correlated anomalous phase diffusion of coupled phononic modes in a sidebanddriven resonator," Nat. Commun. 7, 12694 (2016).

${ }^{10} \mathrm{~W}$. Wustmann and V. Shumeiko, "Parametric resonance in tunable superconducting cavities," Phys. Rev. B 87(18), 184501 (2013).

${ }^{11}$ B. Vlastakis, G. Kirchmair, Z. Leghtas, S. E. Nigg, L. Frunzio, S. M. Girvin, M. Mirrahimi, M. H. Devoret, and R. J. Schoelkopf, "Deterministically encoding quantum information using 100-photon Schrödinger cat states," Science 342(6158), 607 (2013).

${ }^{12}$ S. Puri, S. Boutin, and A. Blais, "Engineering the quantum states of light in a Kerr-nonlinear resonator by two-photon driving," NPJ Quantum Inf. 3(18) (2017).

${ }^{13}$ M. Mirrahimi, Z. Leghtas, V. V. Albert, S. Touzard, R. J. Schoelkopf, L. Jiang, and M. H. Devoret, "Dynamically protected cat-qubits: A new paradigm for universal quantum computation," New J. Phys. 16(4), 045014 (2014).

${ }^{14}$ C. Hayashi, Nonlinear Oscillations in Physical Systems (Princeton, 1985).

${ }^{15}$ D. W. Jordan and P. Smith, Nonlinear Ordinary Differential Equations (Oxford, 2007).

${ }^{16}$ I.-M. Svensson, A. Bengtsson, P. Krantz, J. Bylander, V. Shumeiko, and P. Delsing, "Period-tripling subharmonic oscillations in a driven superconducting resonator," Phys. Rev. B 96(17), 174503 (2017).

${ }^{17}$ Y. Zhang, J. Gosner, S. M. Girvin, J. Ankerhold, and M. I. Dykman, "Time-translation-symmetry breaking in a driven oscillator: From the quantum coherent to the incoherent regime," Phys. Rev. A 96(5), 052124 (2017).

${ }^{18}$ L. Guo, M. Marthaler, and G. Schön, "Phase space crystals: A new way to create a quasienergy band structure," Phys. Rev. Lett. 111, 205303 (2013).

${ }^{19}$ Z. R. Lin, K. Inomata, K. Koshino, W. D. Oliver, Y. Nakamura, J. S. Tsai, and T. Yamamoto, "Josephson parametric phase-locked oscillator and its application to dispersive readout of superconducting qubits," Nat. Commun. 5, 4480 (2014).

${ }^{20}$ I. Mahboob, C. Froitier, and H. Yamaguchi, "A symmetry-breaking electromechanical detector,” Appl. Phys. Lett. 96(21), 213103 (2010). 